\title{
Radyal tabanlı fonksiyon ağlarını kullanarak EKG sinyallerinin sıkıştırılması
}

\author{
Ömer KARAL* \\ Ankara Yıldırım Beyazıt Üniversitesi, Mühendislik ve Doğa Bilimleri Fakültesi, \\ Elektrik-Elektronik Mühendisliği Bölümü, Etlik Batı Yerleşkesi, Ankara. \\ Geliş Tarihi (Recived Date): 07.10.2017 \\ Kabul Tarihi (Accepted Date): 14.03.2018
}

\section{Özet}

Elektrokardiyogram (EKG), kalbin çalışması esnasında kalp kaslarında meydana gelen elektriksel aktivitelerin grafik olarak gösterimidir. EKG, kalp hastalıklarının teşhisinde ve analizinde oldukça önemli bir rol oynamaktadır. Herhangi bir kalp rahatsızlı̆̆ına sahip kişilerin kalbinde meydana gelebilecek bir rahatsızlı̆̆l önceden tespit edebilmek için, EKG sinyalleri sürekli olarak kaydedilir, depolanır ve dijital iletişim ağları üzerinden iletilir. Ancak bu tür kayıtlar ortamdan dolayı gürültüye maruz kalabilir. Dahası, bu şekildeki kayıtlar depolama ve iletimi zorlaştıracak düzeyde büyük miktarda veri üretir. Yukarıda sözü edilen nedenlerden dolay gürültülü ortamda bile etkili bir EKG veri sıkıştırma modeli gereklidir. Bu çalışma, EKG işaretlerinin doğal yapısını gürültülü ortamlarda bile korumak ve daha az sayıda parametre ile yeniden temsil etmek için Radyal Tabanlı Fonksiyon Ağlarını (RTFA) sunar. RTFA'ların tasarımında, modelin yaklaşık doğruluğunu etkileyen önemli unsurlardan birisi olan radyal taban fonksiyonlarının merkezlerinin verimli bir şekilde belirlenmesidir. Bu amaçla, k-means kümeleme algoritması kullanılmıştır. Yeniden yapılandırılmış EKG dalga biçimi, ortalama karesel hata, ortalama mutlak hata ve sıkıştırma oranı açısından niceliksel olarak değerlendirilmiştir. Tüm bu adımlar MATLAB ortamında uygulanmıştır.

Anahtar kelimeler: Elektrokardiogram, radyal tabanlı fonksiyon ağları, veri sıkıştırma.

\section{ECG signal compression using radial basis function networks}

\begin{abstract}
An electrocardiogram (ECG) is the graphical representation of electrical activity in the cardiac muscles of the heart. It plays a significant role in diagnosis and analysis of cardiac diseases. In order to detect any cardiac diseases in advance, the ECG signals
\end{abstract}

\footnotetext{
*Ömer KARAL, karal@ybu.edu.tr, https://orcid.org/0000-0001-8742-8189
} 
are continuously recorded, stored and transmitted over digital communication networks, but such records may be subject to noise due to environment. Moreover, these types of records produce large amounts of data that will make storage and transmission difficult. Due to the reasons mentioned above, an effective ECG data compression model is required even in a noisy environment. This work presents Radial Basis Function Networks (RBFN) to preserve the natural structure of ECG signals even in noisy environments and to re-construct with fewer parameters. In the design of $R B F N$, the center of the radial basis functions, which is one of the important factors affecting the approximate accuracy of the model, is to be determined efficiently. For this purpose, k-means clustering algorithm is used in the paper. The reconstructed ECG waveform was quantitatively evaluated in terms of root mean squared error, mean absolute error, and compression ratio. These steps are implemented in MATLAB environment.

Keywords: Electrocardiogram, radial basis function networks, compression.

\section{Giriş}

Elektrokardiyogram (EKG), kalbin elektriksel aktivitesini kaydetmek için çeşitli sağlık kuruluşlarında yaygın şekilde kullanılan bir yöntemdir. EKG sinyallerinin, hem kalp hastalıklarının izlenmesi hem de uygun tanı ve tedavinin önceden belirlenmesi açısından sürekli kaydedilmesi gerekir. Ancak bu durum, oldukça yüksek miktarda depolama (hafıza) birimini gerektirir. Ayrıca, kaydedilen bu verilerin, dijital iletişim ağlarıyla hızlı bir şekilde iletilmesi de zorlaşır. Söz konusu bu sorunlar, çok büyük miktarlarda veri içeren EKG sinyallerinin (önemli bilgileri korunarak) uygun oranlarda verimli bir şekilde sıkıştırılması ile giderilebilmektedir. EKG işaretlerinin sıkıştırılması modellenmesi ve yeniden elde edilmesine yönelik son dönemde oldukça önemli gelişmeler kaydedilmiştir. EKG işaretlerini sıkıştırmak için kullanılan yöntemler, doğrudan veri sıkıştırma teknikleri, transform (dönüşüm) tabanlı sıkıştırma teknikleri ve öznitelik (parametre) çıkarım tabanlı sıkıştırma teknikleri olmak üzere üç gruba ayrilabilir [1]. i) Doğrudan veri sıkıştırma tekniklerinde [2-3] özgün sinyal, özellikle ilgilenilen bölgeleri, sezgisel belirlenen bazı ölçütlere göre, doğrudan analiz edilerek sıkıştırma yapılır. Bu gruba giren tekniklerin sonuçları genellikle kullanıcıya bağlı olduğu için optimal değildir ve sonuçları kişiden kişiye çeşitlilik gösterir. ii) Öznitelik çıkarım tabanlı tekniklerde [4-5] özgün EKG sinyalinden bazı özel parametrelerin (olasılık dağılımının ölçülmesi gibi) çıkarımına dayanır. Bu parametreler, sıkıştırılmış EKG verisinden özgün sinyali yeniden oluşturmak için kullanılır. Son olarak, iii) dönüşüm tabanlı tekniklerde ise, özgün EKG verileri belirli bir ön işlemden geçirildikten sonra belirlenen dönüşüm fonksiyonuna göre yeni bir uzaya taşınır ve bu uzayda izin verilen hata değerine göre gereğinden fazla olan örnekler ortadan kaldırılarak sıkıştırma yapılır.

Yüksek sıkıştırma oranlarına sahip olmaları ve gürültüye karşı diğer tekniklere göre daha duyarsız olmalarından dolayı, dönüşüm tabanlı yöntemler, uygulamada daha çok tercih edilirler. Bu makalede de dönüşüm tabanlı sıkıştırma kategorisine ait kayıplı yeni bir sıkıştırma tekniği sunulmakta ve değerlendirilmektedir. Fourier dönüşümü (FD) [67], ayrık kosinüs dönüşümü (AKD) [8-9], ve dalgacık dönüşümü (DD) [10-11] gibi yöntemler bu kategoride sayılabilir. 
Son yıllarda, dalgacık tabanlı dönüşüm teknikleri bazı özelliklerinden (zaman frekans lokalizasyonu, enerji sıkıştırması vb.) dolayı EKG verilerinin sıkıştırılmasında yaygın olarak kullanılmıştır [12]. Addison [13], dalgacık tabanlı dönüşü̈m tekniklerinin sürekli ve ayrık zamanda analizini gerçekleştirmiş ve ayrık zaman DD tekniğinin sinyal işleme (filtreleme ve kodlama gibi) yöntemleri ile uyuşan bir matematiğe sahip olduğunu vurgulamıştır. Ayrıca, ayrık DD tabanlı teknikler sürekli DD tabanlı tekniklere göre daha az sayıda dalgacık katsayısı ürettiği için hesaplama süresini de önemli ölçüde azalttığı Addison tarafından gösterilmiştir.

DD tabanlı yöntemlerin performansı, genellikle, ana dalgacık, dalgacık filtrelerinin uzunluğu, ayrışma seviyelerinin sayısı, dalgacık katsayılarının belirlenmesine yönelik yaklaşımlar ve anlam haritasının kodlanmasına bağlıdır. Literatürde DD tabanlı tekniklerin performansını artırmak için genellikle dalgacık katsayılarının daha az sayıda bit ile kodlanmasına yönelik farklı kodlama teknikleri üzerine çalışıldığı görülmektedir. Dalgacık katsayılarını kodlama teknikleri kendi aralarında (i) eşik tabanlı yöntemler [89], (ii) gömülü kodlama tabanlı yöntemler [10-11], (iii) vektör kuantizasyon tabanlı yöntemler [12-13] ve (iv) doğrusal tahmin tabanlı yöntemler [14-15] olmak üzere dört kategoriye ayrılabilir. Bununla beraber, DD tabanlı tekniklerde blok boyutu ile sıkıştırma oranı arasında doğru orantıya sahip bir ilişki vardır. Blok boyutunun arttırılması belirli bir bozulma için sıkıştırma oranını artırır; ancak adaptif dalgacık kodlama şemalarının hesaplama süresi ve hafıza (bellek) gereksinimleri de aynı oranda artacaktır. Bu nedenlerden dolayı, dalgacık dönüşümü ile EKG veri sıkıştırmasında blok boyutuna nasıl karar verileceği konusu hala temel bir problemdir.

Bu çalışma, EKG işaretlerinin sıkıştırılmasında Radyal tabanlı fonksiyon ağları (RTFA) gibi yeni bir dönüşüm yöntemini önerir. Önerilen yöntemin gürültüye karşı gürbüz olması için, kaydedilen özgün EKG verileri Gauss gürültüsüyle bozulmaktadır. Amaç özgün sinyali yeniden oluşturma sırasında gürültülü veride bile en iyi sıkıştırma oranını daha az veri kaybı ile elde etmektir.

Dağınık haldeki veriyi iyi bir şekilde modelleyebilmesinden dolayı [22], RTFA, son zamanlarda, özellikle biyomedikal işaretlerin ve/veya görüntülerin sıkıştırılmasında oldukça ilgi çekmiştir. Perumal ve ark. [23] Huffman, fraktal, Geriye yayılmalı sinir ağları (GYSA), ve RTFA gibi farklı sıkıştırma tekniklerini manyetik rezonans (MR) ve bilgisayarlı tomografi (BT) görüntülerine uygulayarak sıkıştırma oranlarının karşılaştırmasını yapmışlar ve en iyi sonucu RTFA tekniği ile elde etmişlerdir. Yine aynı araştırmacıların bir başka çalışmasında [24], destek vektör makinesi (DVM), RTFA ve GYSA tekniklerini MR, BT ve Pozitron emisyon tomografisi (PET) görüntülerine uygulayarak sıkıştırma oranlarını karşılaştırmışlar, DVM ve RTFA tekniklerinden oldukça iyi sonuçlar almışlardır. Jasmi ve ark. [25], MR ve BT görüntülerinde RTFA ile ayrık DD tekniklerini karşılaştırmış ve RTFA tekniğinin ayrık DD tekniğinden daha iyi sıkıștırma oranı verdiğini gözlemlemişlerdir. RTFA tekniğinin medikal görüntülerindeki başarısından esinlenerek, bu çalışmada, RTFA tekniği iki boyutlu medikal görüntüler yerine bir boyutlu EKG sinyallerinin (filtreleme, kodlama gibi hiçbir ön işlem uygulamadan) doğrudan sıkıştırılmasında kullanılacaktır.

Çalışmanın geri kalan kısmı aşağıdaki gibi düzenlenmiştir. Bölüm 2'de Radyal tabanlı fonksiyon ağları yöntemi genel hatları ile ayrıntılı bir şekilde tanıtılmıştır. Bölüm 3'de EKG verilerinin RTFA ile sıkıştırılması sonucu elde edilen deneysel sonuçlar 
gösterilmiştir. Son bölümde ise uygulamadan elde edilen sonuçlara ilişkin değerlendirmeler yapılmıştır.

\section{Radyal tabanlı fonksiyon ağları}

Radyal tabanlı fonksiyon ağlarının mimari yapısı Şekil 1'de gösterildiği gibi, girdi, çıktı ve gizli katmanlar olmak üzere 3 katmandan oluşur. Girdi katmanı sistemi besleyen girişlerin uygulandığı katmandır. Girdi katmanının çıkışları, doğrudan gizli katmandaki doğrusal olmayan işlem birimlerine (nöronlara) bağlıdır. Gizli katmandaki nöronlar kendine uygulanan girişleri radyal taban fonksiyonundan geçirip sayısal bir değer üretirler. Üretilen bu değerler doğrusal ağırlıklarla çarpılıp çıkış katmanına uygulanır. Çıkış katmanına gelen ağırlıklı girişler toplanarak sayısal bir sonuç üretir.

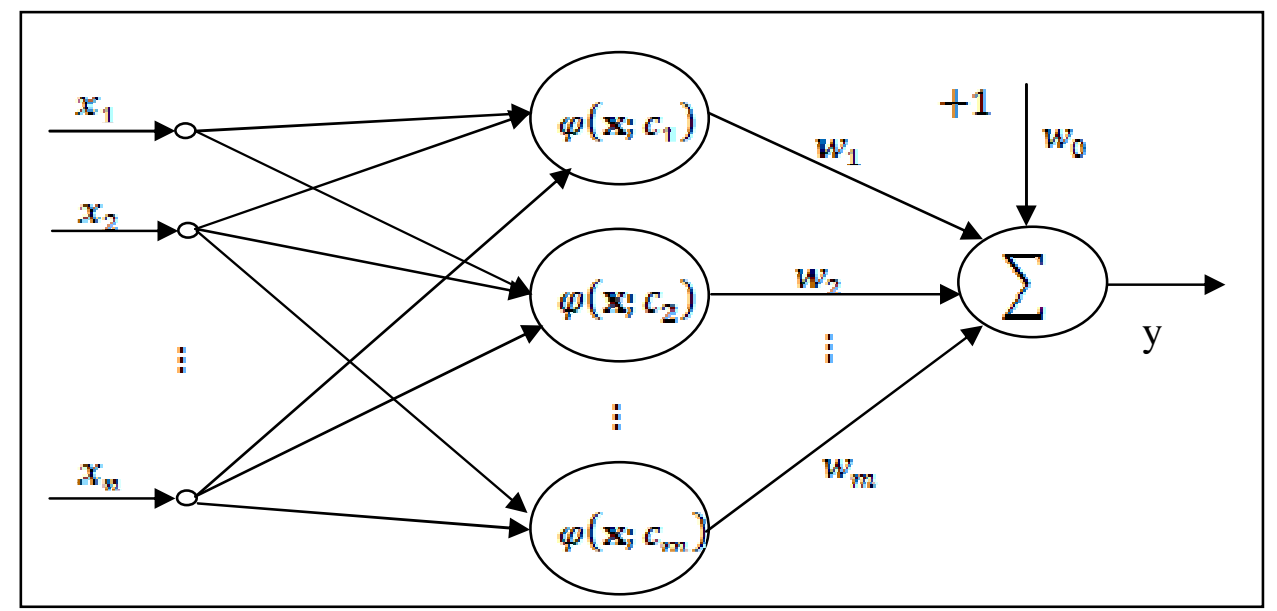

Şekil 1. Radyal tabanlı fonksiyon ağlarının mimari yapısı.

Literatürde en çok tercih edilen radyal taban fonksiyonu Gauss fonksiyonudur ve tanımı aşağıdaki gibi verilir:

$\varphi_{j}\left(\mathbf{x} ; \mathbf{c}_{j}\right)=\exp \left(-\frac{1}{\sigma_{j}^{2}}\left\|\mathbf{x}-\mathbf{c}_{j}\right\|^{2}\right)$

Burada, cj ve $\sigma \mathrm{j}$ sırasıyla nöron $\mathrm{j}$ için radyal taban fonksiyonunun merkezi ve varyansıdır. Buna göre, RTFA'nın çıkışı gizli katmandaki nöronlardan gelen çıkışların ağırlıklı toplamları olarak aşağıdaki gibi elde edilir.

$y=\sum_{j=1}^{m} w_{j} \exp \left(-\frac{1}{\sigma_{j}^{2}}\left\|\mathbf{x}-c_{j}\right\|^{2}\right)+w_{o}$

Verilen bir veri seti $\left\{\left(\mathbf{x}_{s}, y_{s}\right)\right\}_{s=1}^{N} \quad \mathbf{x}_{s} \in R^{\mathrm{n}}$ and $y_{s} \in R$ için doğrusal bağlantı ağırlıkları $w_{j}$ 's aşağıdaki doğrusal cebrik denklem sistemi çözülerek elde edilebilir. 
$\left[\begin{array}{cccc}e^{-\frac{1}{\sigma_{1}^{2}}\left\|\mathbf{x}_{1}-c_{1}\right\|^{2}} & e^{-\frac{1}{\sigma_{2}^{2}\left\|\mathbf{x}_{1}-c_{2}\right\|^{2}}} & \ldots & e^{-\frac{1}{\sigma_{m}^{2}}\left\|\mathbf{x}_{1}-c_{m}\right\|^{2}} \\ e^{-\frac{1}{\sigma_{1}^{2}}\left\|\mathbf{x}_{2}-c_{1}\right\|^{2}} & e^{-\frac{1}{\sigma_{2}^{2}\left\|\mathbf{x}_{2}-c_{2}\right\|^{2}}} & \ldots & e^{-\frac{1}{\sigma_{m}^{2}}\left\|\mathbf{x}_{2}-c_{m}\right\|^{2}} \\ \vdots & \vdots & \ddots & \vdots \\ e^{-\frac{1}{\sigma_{1}^{2}}\left\|\mathbf{x}_{L}-c_{1}\right\|^{2}} & e^{-\frac{1}{\sigma_{2}^{2}\left\|\mathbf{x}_{L}-c_{2}\right\|^{2}}} & \ldots & e^{-\frac{1}{\sigma_{m}^{2}}\left\|\mathbf{x}_{L}-c_{m}\right\|^{2}}\end{array}\right]\left[\begin{array}{c}w_{1} \\ \vdots \\ w_{m}\end{array}\right]+w_{o}\left[\begin{array}{c}1 \\ 1 \\ \vdots \\ 1\end{array}\right]=\left[\begin{array}{c}y_{1} \\ y_{2} \\ \vdots \\ y_{L}\end{array}\right]$

Burada, radyal taban fonksiyonlarının merkezleri çeşitli yöntemlerle bulunabileceği gibi rasgele de atanabilir. Bu çalışmada radyal tabanlı fonksiyonların merkezlerini belirlemek için k-means öbekleme algoritması kullanılmıştır.

Radyal taban fonksiyonlarının varyansları ise genellikle kullanıcı tarafından sağlanır. Eğer sinyal çok girintili çıkıntılı ise (EKG verisinde olduğu gibi) daha düşük varyans değerleri (1'in çok altında), eğer sinyal düzgün bir yapıya sahipse (daha az girintili çıkıntılıysa) daha büyük varyans değerleri (1,2 veya katları) seçilir.

Radyal tabanlı fonksiyon ağı mimarisinde gizli katmanda kaç tane işlem birimi (nöron) bulunacağı önemli bir sorundur. Gizli katmandaki nöron sayısı yeterince arttırılırsa (veri sayısı kadar) tüm verilerden geçen bir model oluşturmak mümkündür. Diğer taraftan gereğinden az sayıda radyal tabanlı fonksiyon kullanılması, girdi ile çıtı arasındaki ilişkiyi anlamlandırmada yetersiz bir model oluşturulmasına yol açacaktır. $\mathrm{Bu}$ bağlamda gizli katmandaki nöron sayısı, RTFA'nın mimarisinde karar verilmesi gereken en önemli parametrelerden birisidir. Genellikle, ya datanın dağılımından esinlenerek yada deneme - yanılma yöntemiyle gizli katmandaki nöron sayısı belirlenir.

\section{EKG sinyalinin radyal tabanlı fonksiyon ağları ile sıkıştırılması ve deneysel sonuçlar}

Önerilen yeni sıkıştırma tekniği RTFA'ya dayanır. RTFA'lar genellikle interpolasyon, yakınlaştırma ve sınıflandırma problemlerinde kullanılır. Park ve Sanberg 1991 yılında, bir gizli katmana sahip RTFA'ların, gizli katmandaki her radyal taban fonksiyonunun varyansı aynı olmak koşuluyla, herhangi bir fonksiyona istenilen oranda yaklaşılabileceğini ispatlamışlardır [26].

RTFA'nın sıkıştırma tekniği olarak kullanılabilmesi için gizli katmanda kullanılan radyal taban fonksiyon sayısının RTFA'da eğitim verisi olarak kullanılan örnek sayısından daha az olması gerekir. Bu durum gereksiz olan eğitim verilerinin eğitimde kullanılmamasına neden olur. Böylece RTFA çıkışında elde edilen sinyal daha az örnek ile temsil edilir ki bu da RTFA'nın girişine uygulanan sinyalin sıkıştırılmış olduğu anlamına gelir.

Bu bölümde gerçekleştirilen tüm deneyler, Intel Core I5 işlemci $3.10 \mathrm{GHz}, 10 \mathrm{~GB}$ RAM ve 64 bit Windows 10 işletim sistemine sahip kişisel bir bilgisayarda kurulu Matlab 2016a ortamında geliştirilen programla (mevcut toolbox kullanılmadan) gerçekleştirilmiştir.

Literatürde, dönüşüm tabanlı tekniklerle yapılan sıkıştırma işlemlerinde [27], sıkıştırma oranı genellikle \%70'in üzerinde olduğu gözlemlenmiştir. Bu çalışmada da benzer sıkıştırma oranını elde edebilmek için gerekli nöron sayısının 35 ile 40 arasında 
seçilmesine karar verilmiştir. Diğer bir deyişle, 35 ile 40 arası radyal taban fonksiyonu kullanılması öngörülmüştür. Ayrıca, EKG sinyallerinin çok düşük genlikte ve girintili çıkıntılı olmasından dolayı sıkıştırma işleminin sonunda özgün sinyalin önemli bilgilerini koruyacak şekilde bir varyans değerine ihtiyaç vardır. Bundan dolayı 0.005 ile 0.01 arasındaki varyans değerleri göz önünde bulundurulmuştur. Sonuç olarak, bu çalışmada, öbek sayısı $(\mathrm{k})$ ve her bir öbekteki radyal taban fonksiyonun varyansı $(\sigma)$ sirasiyla, $\{35,36,37,38,39,40\}$ ve $\{0.005,0.006,0.007,0.008,0.009,0.01\}$ kümelerinden 5 kat çapraz doğrulama tekniği ile $\mathrm{k}=37$, ve $\sigma=0.009$ olarak belirlenmiştir. Tablo 1'den görüleceği üzere, $\sigma=0.009$ varyans değeri için en iyi sonucu (RMSE $=0.060$ ve MAE=0.018) veren öbek sayısı (k) 37'dir yada diğer bir deyişle, Tablo 1'den görüleceği üzere, $\mathrm{k}=37$ öbek için en iyi sonucu ( $\mathrm{RMSE}=0.060$ ve $\mathrm{MAE}=0.018$ ) veren varyans değeri $(\sigma)$ 0.009.'dur.

Tablo 1. Farklı öbek sayıları ve varyanslara göre RTFA'nın RMSE ve MAE değerleri.

\begin{tabular}{|c|c|c|c|c|c|c|c|}
\hline & & & $\mathrm{Var}$ & ans Değerleri & & & \\
\hline & RMSE/MAE & 0.005 & 0.006 & 0.007 & 0.008 & 0.009 & 0.01 \\
\hline & 35 & $0.069 / 0.025$ & $0.115 / 0.035$ & $0.063 / 0.024$ & $0.123 / 0.040$ & $0.095 / 0.030$ & $0.140 / 0.041$ \\
\hline 2 & 36 & $0.066 / 0.022$ & $0.079 / 0.028$ & $0.170 / 0.050$ & $0.104 / 0.031$ & $0.083 / 0.023$ & $0.079 / 0.027$ \\
\hline os & 37 & $0.101 / 0.030$ & $0.118 / 0.033$ & $0.171 / 0.054$ & $0.076 / 0.030$ & $0.060 / 0.018$ & $0.146 / 0.046$ \\
\hline$\ddot{n}$ & 38 & $0.118 / 0.034$ & $0.121 / 0.038$ & $0.158 / 0.046$ & $0.120 / 0.036$ & $0.113 / 0.037$ & $0.067 / 0.020$ \\
\hline 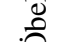 & 39 & $0.077 / 0.026$ & $0.097 / 0.031$ & $0.082 / 0.030$ & $0.103 / 0.033$ & $0.107 / 0.030$ & $0.074 / 0.024$ \\
\hline & 40 & $0.090 / 0.026$ & $0.161 / 0.051$ & $0.141 / 0.039$ & $0.105 / 0.030$ & $0.119 / 0.030$ & $0.137 / 0.041$ \\
\hline
\end{tabular}

Sayısı, merkezi ve varyansı bilinen radyal taban fonksiyonları ile RTFA'nın çatısı oluşturulmuş olur. Artık burada bilinmeyen sadece her bir radyal taban fonksiyonunun katsayısı (ağırlı̆̆ı)'dır. Söz konusu katsayılar ya türev tabanlı herhangi bir yöntemle (türevin ters yönünde adım atılması gibi) yada doğrudan matris tersi alınarak bulunabilir. Bu çalışmada, Eşitlik 3'deki bilinmeyen katsayılar vektörü (w), psedoinverse yöntemi kullanılarak hesaplanmıştır.

Önerilen yöntemin başarımını ölçmek için RTFA çıkışında elde edilen sinyalin RTFA'nın girişine uygulanan (özgün) sinyale ne kadar benzediği (aradaki hata) ve sıkıştırma oranı incelenmiştir.

RTFA'nın sıkıştırma performansı 2 farklı hata ölçütü ile hesaplanmıştır. Bunlar sirasıyla, ortalama karesel hatanın kökü (Root Mean Square Error=RMSE) ve ortalama mutlak hata (Mean Absolute Error=MAE)'dır ve tanımları aşağıdaki gibi verilir.

$$
\begin{aligned}
& R M S E=\sqrt{\frac{1}{N} \sum_{s=1}^{N}\left(y_{s}-f\left(\mathbf{x}_{s}\right)\right)^{2}} \\
& M A E=\frac{1}{N} \sum_{s=1}^{N}\left|\left(y_{s}-f\left(\mathbf{x}_{s}\right) / y_{s}\right)\right|
\end{aligned}
$$

Burada, $\mathrm{N}$ toplam eğitim verisini (bu çalışma için 268), $y_{\mathrm{s}}$ ve $f\left(\mathbf{x}_{\mathrm{s}}\right)$ sırasıyla özgün sinyalde $\mathbf{x}_{\mathrm{s}}$ 'e karşılık gelen ve RTFA çıkışında elde edilen çıkışları gösterir.

Sıkıştırma oranı (SO) için aşağıdaki ifade kullanılabilir. 
$\mathrm{SO}=\frac{\text { Özgün sinyal için gerekli parametre sayısı }}{\text { Sıkıştırılmış sinyal için gerekli parametre sayısı }}$

Sıkıştırılmış sinyal için gerekli parametre sayısı, RTFA'nın çıkışında elde edilen sinyalde kullanılan parametre sayısına denktir. Diğer bir deyişle, radyal taban fonksiyonlarının ağırlıklı toplamından elde edilen parametre sayısıdır.

Şekil 2'de MIT-BIH veri tabanından alınan normal bir atımlık (sinüs ritmi) EKG işareti (lacivert) gösterilmektedir. Bu EKG verisi 268 örnekle temsil edilmekte ve örnekleme frekans1 $360 \mathrm{~Hz}$ 'dir ve her bir örnek 12 bit ile temsil edilmektedir. Şekil 2'den görüleceği üzere, 1 atımlık EKG sinyali $\mathrm{P}, \mathrm{QRS}$ ve T olarak adlandırılan üç farklı dalga biçiminden oluşur. Dalgalar arasındaki mesafeler, dalgaların süresi, dalgaların yükseklikleri, dalgaların şekilleri, dalgaların düzenli olarak birbirlerini takip ederken meydana gelebilecek değişiklikler kalpte olabilecek yapı değişikliğini veya hastalığı gösterebilir. Önerilen yöntemin gürültüye karşı gürbüz (robust) olduğunu göstermek için, özgün EKG verilerine ortalaması sifır $(\mu=0)$ olan altı farklı varyansta $\{\tau=0.001$, $\tau=0.003, \tau=0.005, \tau=0.01, \tau=0.03, \tau=0.05\}$ Gauss gürültüsü $(1 / \sqrt{2 \pi}) \exp \left(-(x-\mu)^{2} / 2 \tau^{2}\right)$ eklenmiştir. Şekil 2'deki pembe noktalar ( $\mu=0$ ve $\tau=0.001$ için) gürültülü verileri göstermektedir. Burada yatay eksen zamanı (mili saniye) düş̧ey eksen genliği (mili volt) ifade eder.

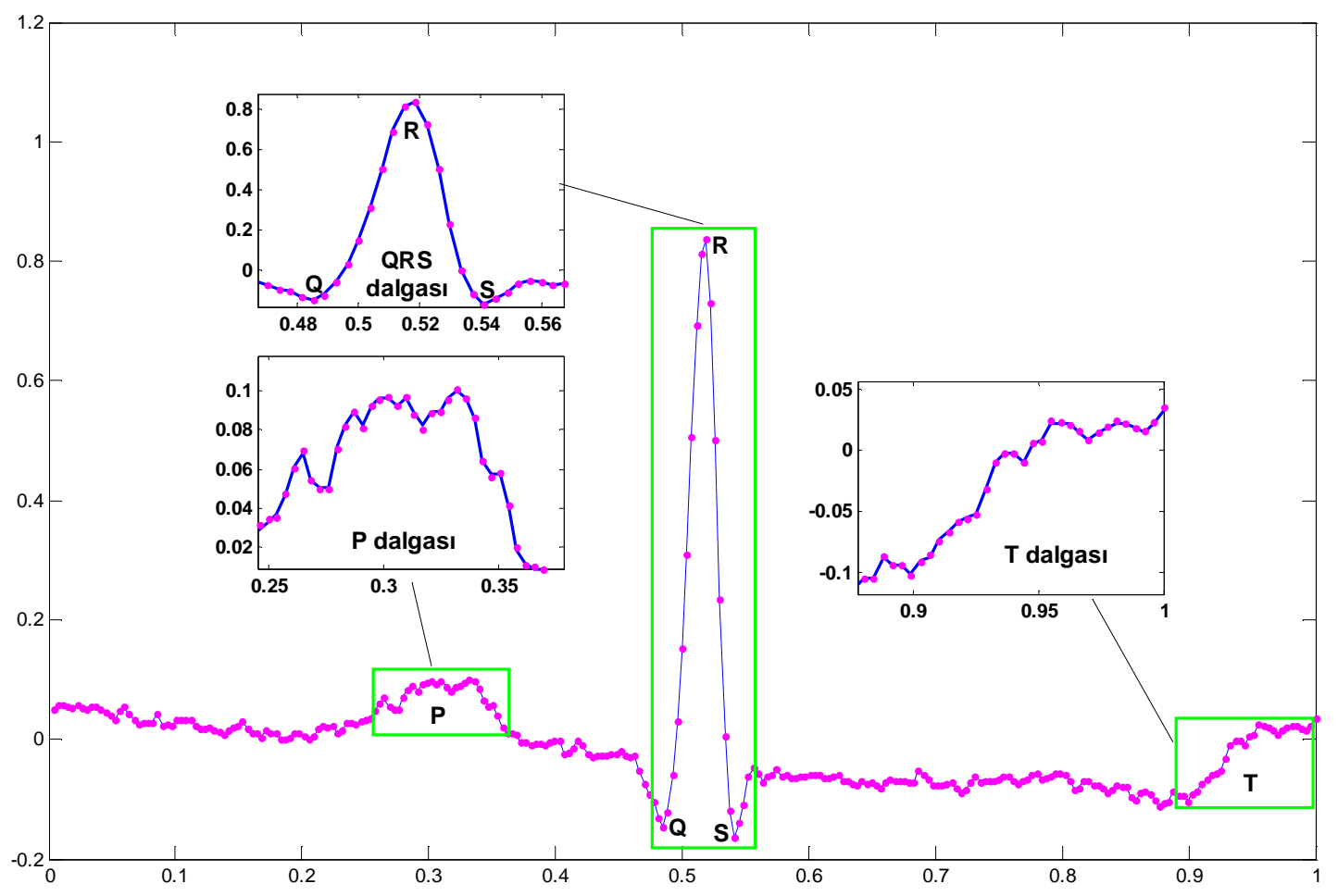

Şekil 2. Özgün EKG (lacivert) sinyali, Gauss gürültüsü eklenmiş EKG (açık mor) sinyali ve Özgün EKG sinyalini oluşturan $\mathrm{P}$, QRS ve T dalga biçimleri (yeşil dikdörtgenler).

Gerçekleştirilen simülasyon sonucunda, özgün EKG sinyali (kırmızı), Gauss gürültü ( $\mu=0$ ve $\tau=0.03$ ) ile bozulmuş EKG sinyali (yeşil) ve RTFA ( $\mathrm{k}=37$ ve $\sigma=0.009$ ) ile sıkıștırılmış gürültülü EKG sinyali (lacivert) Şekil 3'de bir arada gösterilmiștir. 


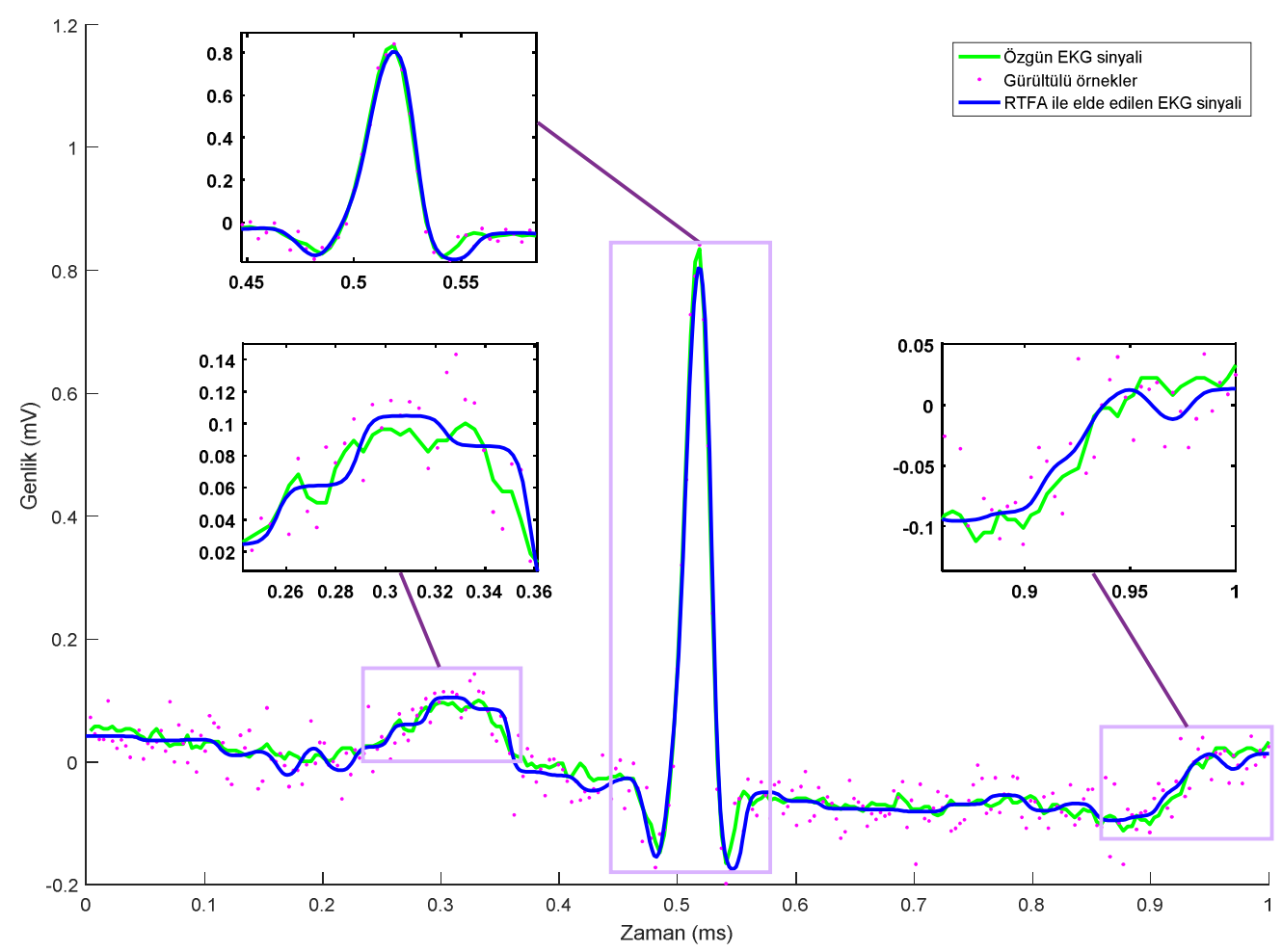

Şekil 3. Özgün (yeşil) ve Gauss gürültü ( $\mu=0$ ve $\tau=0.03$ ) ile bozulmuş (pembe noktalar) ve RTFA ( $\mathrm{k}=37$ ve $\sigma=0.009$ ) ile elde edilmiş EKG sinyalleri (lacivert).

$\mathrm{RMSE}=0.155, \mathrm{MAE}=0.061$ ve $\mathrm{SO}=3.62$.

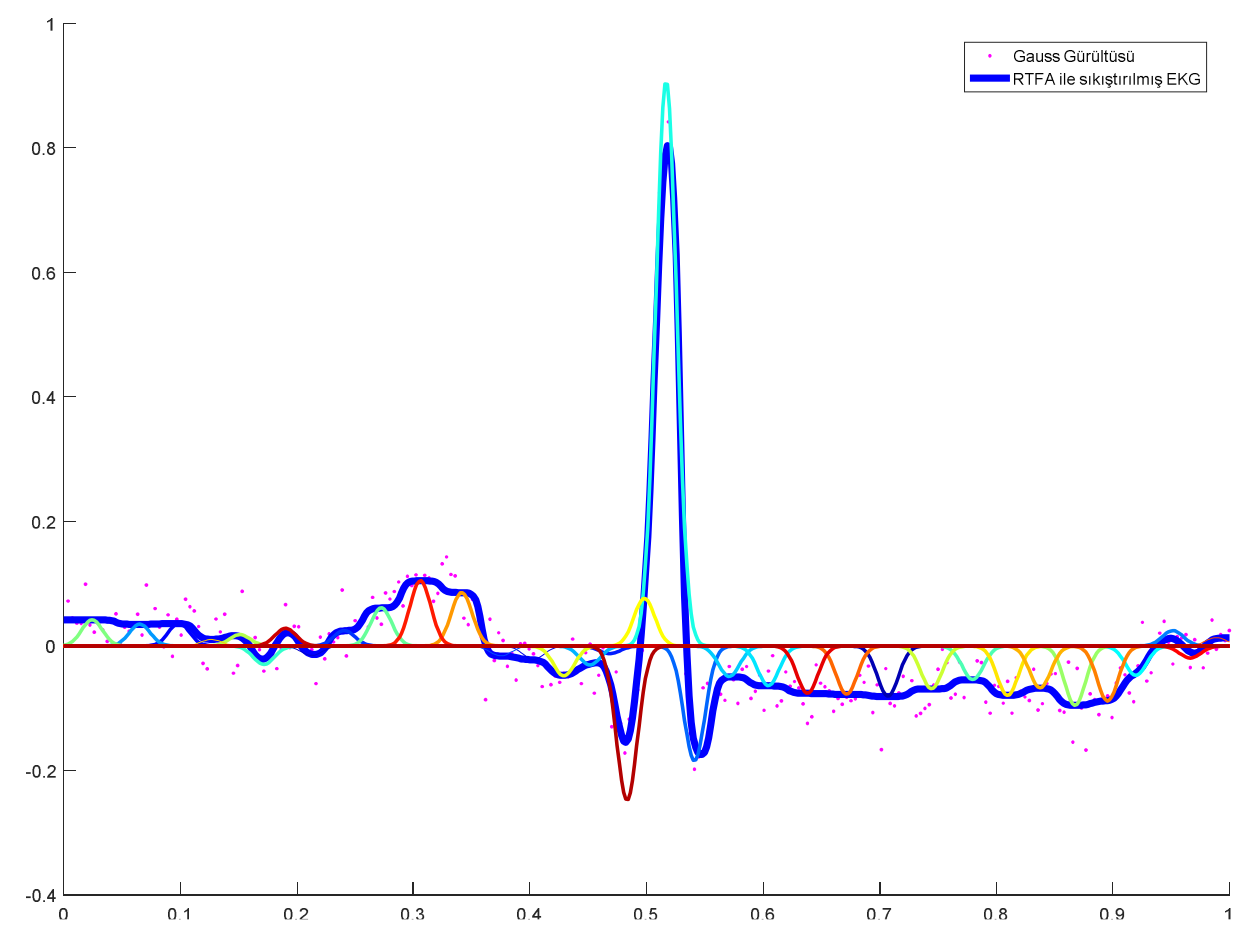

Şekil 4. EKG sinyalinin RTFA ile sıkıştırılmasında kullanılan radyal taban fonksiyonlarının (37 adet) genişliklerinin, büyüklerinin ve merkezlerinin farklı renklerle gösterimi. 
Şekil 3'deki RTFA ( $\mathrm{k}=37$ ve $\sigma=0.009$ ) ile sıkıştırılmış EKG sinyali için 37 radyal taban fonksiyonu kullanılmıştır. Sıkıştırma oranı, RTFA'nın çıkışında elde edilen sıkıştırılmış fonksiyon için gerekli parametre sayısı, yani kullanılan radyal fonksiyon sayısından hesaplanabilir. Varyansı $(\sigma=0.009)$ aynı olduğundan, sadece 2 parametre (büyüklük ve merkez) ile temsil edilen her bir radyal taban fonksiyonunu tanımlamak için, 37x2=74 parametreye ihtiyaç vardır. Böylece özgün EKG sinyali için gerekli 268 eğitim örneği (parametre) yerine sadece 74 parametreye sahip 37 nöronlu RTFA ile yapılan sıkıştırmanın oranı: $\mathrm{SO}=268 / 74=3,62$ olarak bulunur. RTFA ile sıkıștırılmış EKG (lacivert) sinyalini oluşturan her bir radyal taban fonksiyonunun genişlik (variance), büyüklük (amplitude) ve merkez (center) bilgileri farklı renklerde Şekil 4'de gösterildiği gibidir. Her bir renk, bir radyal taban fonksiyonunu ifade eder.

Aynı sıkıştırma oranında $(\mathrm{SO}=3,62)$ farklı gürültü $(\tau)$ değerleri için, RTFA'nın performansı (RMSE ve MAE gibi ayrıntılı analiz sonuçları) Tablo 2'de listelenmiştir.

Tablo 2. Farklı varyansa sahip gürültü dağılımları altında RTFA' dan elde edilen RMSE ve MAE değerleri.

\begin{tabular}{|l|c|c|}
\hline Gürültü Varyans1 & RMSE & MAE \\
\hline $\boldsymbol{\tau}=\mathbf{0 . 0 0 1}$ & $\mathbf{0 . 0 6 0}$ & $\mathbf{0 . 0 1 8}$ \\
\hline$\tau=0.003$ & 0.109 & 0.033 \\
\hline$\tau=0.005$ & 0.166 & 0.053 \\
\hline$\tau=0.01$ & 0.175 & 0.053 \\
\hline$\tau=0.03$ & 0.155 & 0.061 \\
\hline$\tau=0.05$ & 0.146 & 0.068 \\
\hline
\end{tabular}

RTFA ile sıkıştırma işleminde dikkat edilmesi gereken en önemli unsur radyal taban fonksiyonlarının yerinin (merkezlerinin) en uygun şekilde belirlenmesidir. $\mathrm{Bu}$ çalışmada k-means öbekleme algoritması kullanılmıştır. Ancak, k-means öbekleme yönteminde merkezler rasgele atanır ve optimal yeri Öklid mesafesi hesaplanarak bulunur. Elde edilen merkezler verinin dağılımı ile tam örtüşmeyebilir. Bundan dolayı, öbeklere ayrilacak veriyi uygun şekilde temsile edecek merkezlerin belirlenmesinde kmeans algoritmasının birkaç kez çalıştırılması gerekebilir. Bu durum, RTFA'nın başarısını daha da artıracaktır.

\section{Sonuçlar}

Radyal tabanlı fonksiyon ağları (RTFA) interpolasyon, yakınlaştırma ve sınıflandırma gibi birbirinden farklı problemleri çözmek için birçok uygulamada yaygın olarak kullanılmaktadır.

$\mathrm{Bu}$ çalışmada, EKG işaretlerinin doğal yapısını (P, QRS ve $\mathrm{T}$ dalga biçimlerini) gürültülü ortamlarda bile bozmadan korumak ve daha az sayıda parametre kullanılarak yeniden oluşturulmasını sağlamak için RTFA yöntemi önerilmiştir. Yöntemin temel yaklaşımı, RTFA'nın gizli katmanında kullanılan ve radyal taban fonksiyonunu tanımlayan parametre sayısının eğitim verisi olarak kullanılan örnek sayısından (özgün EKG verisinden) daha az olacak şekilde seçilmesiyle gürültülü ortamlarda bile etkili bir sıkıştırma işlemi gerçekleştirmektir. RTFA'ların tasarımında, modelin yaklaşık doğruluğunu etkileyen önemli unsurlardan birisi olan radyal taban fonksiyonlarının 
merkezlerinin verimli bir şekilde belirlenmesidir. Bu amaçla, k-means öbekleme algoritması kullanılmıştır. k-means öbekleme algoritmasında merkezler rastgele atanır ve optimal yeri Öklid mesafesine göre belirlenir. Bundan dolayı algoritmanın birkaç kez çalıştırılıp en uygun yerin tespit edilmesi gerekebilir.

Gerçekleştirilen bilgisayar simülasyonlarında, uygun sayıda radyal taban fonksiyonu seçerek (37 nöron), önerilen yöntemle RMSE=0.060, MAE=0.018 ve 3.62 sıkıştırma oranı elde edilmiştir ki bu sonuç literatürde mevcut diğer dönüşüm tabanlı yöntemlerle elde edilen sonuçlara oldukça benzerdir. Sonuç olarak, önerilen yöntem EKG işaretlerinin sıkıştırılmasında önemli bir seçenek oluşturmaktadır. Dahası önerilen yöntem, farklı radyal taban fonksiyonlarıyla daha ileri çalışmalara genişletilebilir.

\section{Kaynaklar}

[1] Jalaleddine, S.M., Hutchens, C.G., Strattan, R.D. ve Coberly, W.A., ECG data compression techniques-a unified approach, IEEE Transsctions on Biomedical Engineering, 37(4), 329-343, (1990).

[2] Ishijima, M., Shin, S.B., Hostetter, G.H. ve Sklansky, J., Scan-along polygonal approximation for data compression of electrocardiograms, IEEE Transactions on Biomedical Engineering, 11, 723-729, (1983).

[3] Horspool, R.N. ve Windels, W.J., ECG compression using Ziv-Lempel techniques, Computers and biomedical research, 28(1), 67-86, (1995).

[4] Imai, H., Kiraura, N. ve Yoshlda, Y, An efficient encoding method for electrocardiography using spline functions, Systems and Computers in Japan, 16(3), 85-94, (1985).

[5] Barlas, G.D. ve Skordalakis, E.S., A novel family of compression algorithms for ECG and other semiperiodical, one-dimensional, biomedical signals, IEEE transactions on biomedical engineering, 43(8), 820-828, (1996).

[6] Reddy, B.S. ve Murthy, I.S.N., ECG data compression using Fourier descriptors, IEEE Transactions on Biomedical Engineering, 4, 428-434, (1986).

[7] Al-Nashash, H.A.M., ECG data compression using adaptive Fourier coefficients estimation, Medical engineering \& physics, 16, 1, 62-66, (1994).

[8] Benzid, R., Messaoudi, A. ve Boussaad, A., Constrained ECG compression algorithm using the block-based discrete cosine transform, Digital Signal Processing,18(1), 56-64, (2008).

[9] Bendifallah, A., Benzid, R. ve Boulemden, M., Improved ECG compression method using discrete cosine transform. Electronics letters, 47(2), 87-89, (2011).

[10] Chen, J., Itoh, S. ve Hashimoto, T., ECG data compression by using wavelet transform, IEICE Transactions on Information and Systems, 76, 12, 14541461, (1993).

[11] Patel, S. ve Datar, A., ECG data compression using wavelet transform. International Journal of Engineering Trends \& Technology, 10, 770-776, (2014).

[12] Manikandan, M.S. ve Dandapat, S., Wavelet-based electrocardiogram signal compression methods and their performances: a prospective review, Biomedical Signal Processing and Control, 14, 73-107, (2014).

[13] Addison, P.S., Wavelet transforms and the ECG: a review, Physiological Measurement, 26(5), R155, (2005). 
[14] Abo-Zahhad, M., Ahmed, S.M., Sabor, N. ve Al-Ajlouni, A.F., Wavelet threshold based ECG data compression technique using immune optimization algorithm, International Journal of Signal Processing, Image Processing and Pattern Recognition, 8(2), 307-360, (2015).

[15] Swarnkar, A., Kumar, R., Kumar, A. ve Khanna, P., Performance of different threshold function for ECG compression using Slantlet transform, Proceedings, $4^{\text {th }}$ International Conference on Signal Processing and Integrated Networks, 375-379, Noida, India, (2017).

[16] Ballesteros, D.M., Moreno, D.M. ve Gaona, A.E., FPGA compression of ECG signals by using modified convolution scheme of the Discrete Wavelet Transform, Ingeniare, Revista chilena de ingeniería, 20, 1, (2012).

[17] Al-Busaidi, A.M., Khriji, L., Touati, F., Rasid, M.F.A. ve Mnaouer, A B., Realtime DWT-based compression for wearable electrocardiogram monitoring system, Proceedings, IEEE 8th GCC Conference and Exhibition (GCCC), 1-6, Muscat, Umman, (2015).

[18] Huang, B., Wang, Y. ve Chen, J., ECG compression using the context modeling arithmetic coding with dynamic learning vector-scalar quantization, Biomedical Signal Processing and Control, 8(1), 59-65, (2013).

[19] Hung, K. C., Wu, T.C., Lee, H.W. ve Liu, T.K., EP-based wavelet coefficient quantization for linear distortion ECG data compression, Medical Engineering \& Physics, 36(7), 809-821, (2014).

[20] Ramakrishnan, A.G. ve Saha, S., ECG coding by wavelet-based linear prediction, IEEE Transactions on Biomedical Engineering, 44, 12, 1253-1261, (1997).

[21] Al-Shrouf, A., Abo-Zahhad, M. ve Ahmed, S.M., A novel compression algorithm for electrocardiogram signals based on the linear prediction of the wavelet coefficients, Digital Signal Processing, 13, 4, 604-622, (2003).

[22] Le Gia, Q.T. ve Wendland, H., Data compression on the sphere using multiscale radial basis function approximation, Advances in Computational Mathematics, 40(4), 923-943, (2014).

[23] Balasubramani, P. ve Murugan, P. R. Efficient image compression techniques for compressing multimodal medical images using neural network radial basis function approach. International Journal of Imaging Systems and Technology, 25(2), 115-122, (2015).

[24] Perumal, B., Rajasekaran, M.P. ve Murugan, H., Comparison of neural network algorithms in image compression technique. Proceedings, $3^{\text {rd }}$ International Conference on Emerging Technological Trends (ICETT), Kerala, India, 1-6, (2016).

[25] Jasmi, R.P., Perumal, B. ve Rajasekaran, M.P., Comparison of medical image compression using DWT algorithm and neural network techniques. Advances in Natural and Applied Sciences, 8, 19, 1-10, (2014).

[26] Park, J. ve Sandberg, I.W., Universal approximation using radial-basis-function networks, Neural computation, 3(2), 246-257, (1991).

[27] Ranjeet, K., Kumar, A. ve Pandey, R.K., ECG signal compression using different techniques. Advances in Computing, Communication and Control, 231-241, (2011). 